\title{
Analysis of Serum Protein Precipitated with Antiserum by Matrix-Assisted Laser Desorption Ionization / Time-of-Flight and Electrospray Ionization Mass Spectrometry as a Clinical Laboratory Test
}

\author{
Toyofumi Nakanishi and Akira Shimizu \\ Department of Clinical Pathology, Osaka Medical College, Takatsuki, Osaka, 569, Japan
}

\section{Nobuhiko Okamoto}

Department of Planning and Research, Osaka Medical Center and Research Institute for Maternal and Child Health, 840 Murodo-cho, Izumi, Osaka, 590-02, Japan

\author{
Arnd Ingendoh and Michiko Kanai \\ Finnigan MAT Instruments, Inc., Nishi-Shinjuku Toyokuni Building, 2-5-8 Hatsudai, Shibuya-ku, Tokyo, 151, \\ Japan
}

\begin{abstract}
Serum transferrin precipitated with specific antisera was analyzed by matrix-assisted laser desorption ionization/time-of-flight mass spectrometry (MALDI/TOF-MS) and electrospray ionization-mass spectrometry (ESI-MS). When analyzed by MALDI, transferrin showed signal peaks that clearly could be separated from ions of IgG present in the immunoprecipitate. By ESI-MS, when the immunoprecipitates were loaded through a microcapillary polymeric reversed-phase column connected to the electrospray ionization probe, the mass spectra of transferrin were observed with a high signal-to-noise ratio and good resolution. By MALDI/TOF-MS, the observed molecular weight of normal transferrin was $\sim 1.2 \mathrm{ku}$ smaller when analyzed in the reflectron mode than in the linear mode. The observed molecular weight of transferrin treated with sialidase was approximately the same in both modes. A comparison between the results obtained in both modes may help to estimate the number of sialic acids on the protein molecule. A transferrin isoform with a molecular weight of $\sim 2.2 \mathrm{ku}$ less than the normal species was identified in the serum of patients with a carbohydrate-deficient glycoprotein syndrome as well as in heavy alcohol consumers. ( $\mathrm{Am}$ Soc Mass Spectrom 1995, 6, 854-859)
\end{abstract}

$\mathrm{R}$ ecent developments in mass spectrometry with matrix-assisted laser desorption ionization (MALDI) and electrospray ionization (ESI) allow the accurate molecular mass determination of proteins at the low picomole level [1, 2]. For clinical purposes protein variants have been identified by electrophoresis, but variants with the same electric charge as the native protein cannot be identified. Identification of the variants usually requires a complex and timeconsuming type of chemical analysis. However, by use of the above-mentioned recent mass spectrometry methods, identification is possible directly with the intact macromolecule. To apply these strategies for clinical diagnosis, simple and reliable methods of sample preparation are desired.

Address reprint requests to Dr. Akira Shimizu, Department of Clinjcal Pathology, Osaka Medical College, 2-7, Daigakumachi, Takatsuki-city, Osaka, 569, Japan.
As the first test for the accessibility of such mass spectrometry methods for clinical diagnosis, an abnormal transferrin isoform, which is present in the serum of patients with carbohydrate-deficient glycoprotein (CDG) syndrome [3] as well as in high alcohol consumers $[4,5]$, was analyzed by ESI and MALDI. The syndrome, a multisystem disorder that affects the nervous system, was first reported by Jaeken et al. [3]. The biochemical feature of the syndrome is an abnormal carbohydrate structure of glycoproteins. Normal transferrin mainly has a single isoform that contains two asparagine $N$-linked disialylated biantennary carbohydrate chains. Wada et al. [6] and Yamashita et al. $[7,8]$ reported that the abnormal species of CDG syndrome transferrin lack either or both of these disialylated biantennary carbohydrate chains, as judged by conventional chemical methods [7] and by electrospray ionization mass spectrometry (ESI-MS) [6, 8]. Nakanishi et al. [9] and Wada et al. [10] also confirmed the molecu- 
lar weight difference by MALDI. The transferrin from CDG patients consists of an isoform of normal size and two isoforms with a 2.2 and $4.4 \mathrm{ku}$ lower molecular weight.

The most common method to prepare serum proteins for such mass spectrometry analysis used to be affinity chromatography with an immobilized specific antibody. In a preliminary report, we described a new method that is suitable for clinical laboratory tests [9], whereby serum transferrin $(\sim 300 \mathrm{mg} / \mathrm{dL}, 38 \mathrm{nM}$ in serum) was precipitated with specific antisera and analyzed by MALDI. Ion signals of the transferrin and the antibody included in the immunoprecipitate could be separated clearly and were not superimposed in the obtained mass spectra. In the present work we analyzed the serum proteins precipitated similarly with a specific antibody by ESI-MS that is expected to allow a more precise analysis. Instead of conventional infusion sample loading methods, we loaded the immunoprecipitate through a microcapillary polymeric reversedphase column connected to the ESI probe.

For glycopeptides that incorporate sialic acids, ions generated by MALDI are extremely unstable and split off the sialic acids after a certain lifetime [11, 12]. To investigate this "metastability" for a large glycoprotein with a greater degree of freedom like transferrin, we have analyzed native and sialidase-treated transferrin ions in different modes of operation of the time-offlight mass spectrometer. Additionally, the carbohydrate-deficient transferrin was studied again by MALDI with special attention to the sialic acids.

\section{Experimental}

\section{Materials}

Serum was obtained from normal healthy persons, from two patients with CDG syndrome in the same family [13] and from heavy alcohol drinkers, who consumed more than $100-\mathrm{g}$ alcohol per day. The venous blood obtained without anticoagulant was allowed to stand at room temperature until it clotted and then was centrifuged at $2100 \mathrm{~g}$ for $10 \mathrm{~min}$. The supernatant was obtained.

Transferrin was prepared by affinity chromatography [7] and the immunoprecipitation method. The immunoprecipitation method was described in a previous report [9]. Briefly, test serum was mixed with antitransferrin serum (polyclonal rabbit serum purchased from Hoechst Japan Ltd. Tokyo; Lot No. 153443B) and ethylenediaminetetraacetic acid (EDTA) was added to give a final concentration of $2.5 \mathrm{mM}$; the serum was kept at $4{ }^{\circ} \mathrm{C}$ overnight. The precipitate was centrifuged and washed three times with saline followed by five washes with distilled water. The precipitate was then dissolved in methanol-water (1:1) that contained $1 \%$ acetic acid. The serum concentration of transferrin was estimated by a nephelometric assay with an antitransferrin antibody. The transferrin content in serum was $\sim 300 \mathrm{mg} / \mathrm{dL}$, which is $\sim 4 \%$ of total protein and is $\sim 1 / 16$ of albumin. The required ratio of test serum and antiserum is dependent on the titer of antiserum. There is a broad acceptable range to this ratio to allow mass spectrometric detection of the target protein. With the lot of antiserum we used in the present experiment, good ion peaks of the transferrin were observed at a ratio of test serum to antiserum of 1:1. The amount of test serum and antiserum could be reduced. When we used $50 \mu \mathrm{L}$ of each serum, $1 / 400$ of the precipitate was applied on the time-offlight (TOF) mass spectrometer and $1 / 30$ on the microcapillary liquid chromatography (LC) ESI mass spectrometer. Commercially purchased transferrin (Sigma Chemical Co., St. Louis, MO) also was used.

\section{Mass Spectrometry and Microcapillary High Performance Liquid Chromatography}

MALDI/TOF mass spectra were recorded on a JMSLDI 1700 (linear mode; JEOL Ltd., Tokyo, Japan) and a Vision 2000 (reflectron and linear type; Finnigan MAT, Bremen, Germany). Detailed descriptions of the instruments can be found elsewhere [14, 15]. The matrices used were 2,5-dihydroxybenzoic acid and sinapinic acid (Aldrich Chemical Co., Milwaukee, WI). The applied amount of protein sample for each analysis was 0.5-2 pmol.

ESI mass spectra were obtained on a TSQ-7000 triple quadrupole mass spectrometer (Finnigan MAT, San Jose, CA) equipped with an electrospray ion source and a polymeric reversed-phase microcapillary high performance liquid chromatography (HPLC) column (PLRP-S, $1 \mathrm{~mm} \times 50 \mathrm{~mm}, 1000 \AA, 8 \mu \mathrm{m}$; Michrom Bioresources Inc., Pleasanton, CA). The proteins in the immunoprecipitates were eluted with a gradient of $0.2-86 \%$ acetonitrile containing $2 \%$ acetic acid over 15 min at a flow rate of $50 \mu \mathrm{L} / \mathrm{min}$. In some cases, methanol was used instead of acetonitrile. The applied amount of transferrin for each analysis was $10 \mu \mathrm{L}$ of $\sim 20$-pmol $/ \mu \mathrm{L}$ solution. The signal peaks were detected by using a cell inserted between the column and the mass spectrometer. ESI scanning was performed $m / z 2000$ to 4000 in $5 \mathrm{~s}$ for a high mass range and $m / z$ 500 to 2500 in $5 \mathrm{~s}$ for a low mass range. Calibration was performed by using the multiply-charged ions from a separate introduction of horse heart myoglobin and hen eggwhite lysozyme.

Transferrin was treated with sialidase (Sigma Chemical Co.) in 0.05-M citrate buffer, pH 5.5, for $24 \mathrm{~h}$ at an enzyme to transferrin ratio of $4 \mathrm{mu} / \mathrm{nmol}$.

\section{Results}

Figure 1 shows the analysis of transferrin prepared from a healthy individual (Figure 1a) and from a patient with CDG (Figure 1b) by immunoprecipitation via ESI-MS connected with a microcapillary HPLC. Figure insets represent chromatograms of the separation of the transferrin-antibody mixture by HPLC. In 


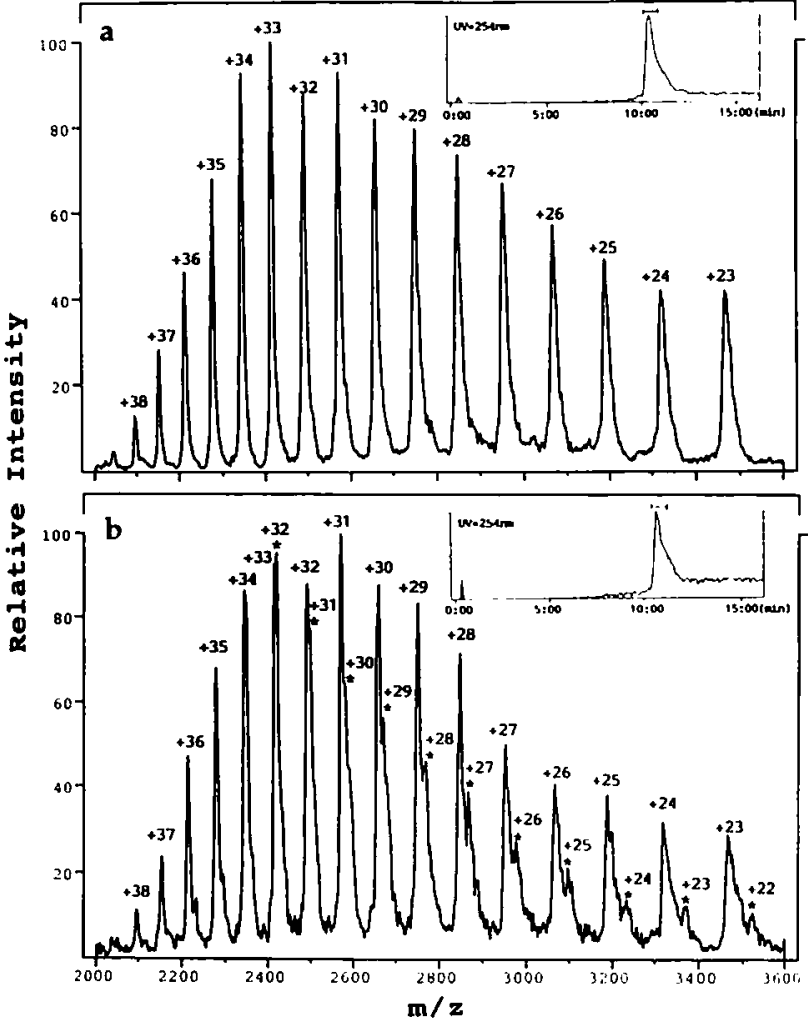

the corresponding mass spectra of the peaks (marked by horizontal bars), the signals of the different multiply charged transferrin ions were resolved clearly (Figure $1 \mathrm{a}$ and $\mathrm{b}$ ). Multiply charged ion peaks of the main component yielded reasonable molecular weight estimations of normal transferrin in both spectra (Table 1). In the spectra of patient transferrin (Figure 1b) a series of ion peaks of the subcomponent (marked by asterisks) were observed between the ion peaks of the main component. The spectra of a normal control (Figure 1a) showed no such subcomponent. Figure 2 shows sections of MALDI/TOF (LDI-1700) mass spectra in the
Figure 1. Analysis of transferrin prepared by immunoprecipitation via ESI-MS connected to a microcapillary HPLC. Chromatograms monitored by UV absorption at $254 \mathrm{~nm}$ are presented in the insets. Mass spectra of the peaks, marked by horizontal bars on the chromatograms, show the multiply charged ions of transferrin. Spectra are from a healthy control (a) and from a patient with CDG (b). Analyses used a Finnigan MAT TSQ-7000 triple quadrupole mass spectrometer coupled with an ultrafast microprotein analyzer. Column: PLPR-S $(1000 \AA, 8 \mu \mathrm{m}, 1 \mathrm{~mm} \times$ $50 \mathrm{~mm}$ ); flow rate: $50 \mu \mathrm{L} / \mathrm{min}$; linear gradient from $10 \%$ B to $95 \% \mathrm{~B}$ in $15 \mathrm{~min} . \mathrm{A}=$ methanol-water $(2: 98)$ and $2 \%$ acetic acid $B=$ methanol-water $(90: 10)$ and $2 \%$ acetic acid.

Table 1. Observed molecular weights of intact, desialylated, and carbohydrate-deficient transferrin measured by MALDI/TOF and ESI mass spectrometry and theoretical molecular weights of each form

\begin{tabular}{|c|c|c|c|c|}
\hline & Intact normal & Desial. normal & Difference $^{a}$ & Intact CDG \\
\hline Theor. MW & $79,555.26$ & $78,390.18$ & 1165.08 & $77,349.08$ \\
\hline TSQ-7000 (ESI) & $79,557^{b}$ & 78,384 & 1173 & 77,354 \\
\hline $\begin{array}{l}\text { Vision } 2000^{c} \\
\text { (MALDI reflectron) }\end{array}$ & $\begin{array}{c}78,535 \pm 127 \\
(3)\end{array}$ & $\begin{array}{c}78,483 \pm 30 \\
\text { (3) }\end{array}$ & 52 & 76,693 \\
\hline $\begin{array}{l}\text { JMS-LDI } 1700^{\circ} \\
\text { (MALDI linear) }\end{array}$ & $\begin{array}{c}79.710 \pm 300 \\
(5)\end{array}$ & $\begin{array}{c}78,442 \pm 230 \\
\text { (3) }\end{array}$ & 1268 & $\begin{array}{c}77,485 \pm 260 \\
(5)\end{array}$ \\
\hline \multicolumn{5}{|l|}{ Difference } \\
\hline $\begin{array}{l}\text { between linear } \\
\text { and reflectron }\end{array}$ & 1.175 & -41 & & 792 \\
\hline
\end{tabular}

${ }^{a}$ Difference between molecular weight of intact and desialylated normal transferrin (intact - desialylated).

${ }^{b}$ Standard deviation of each analysis by TSQ-7000 was less than 10.

c Each value is the mean value and standard deviation of analyses. The number of measurements is shown in parentheses.

dDifference between molecular weight observed by linear mode and reflectron mode MALDI/TOF mass spectrometry (linear - reflectron). 


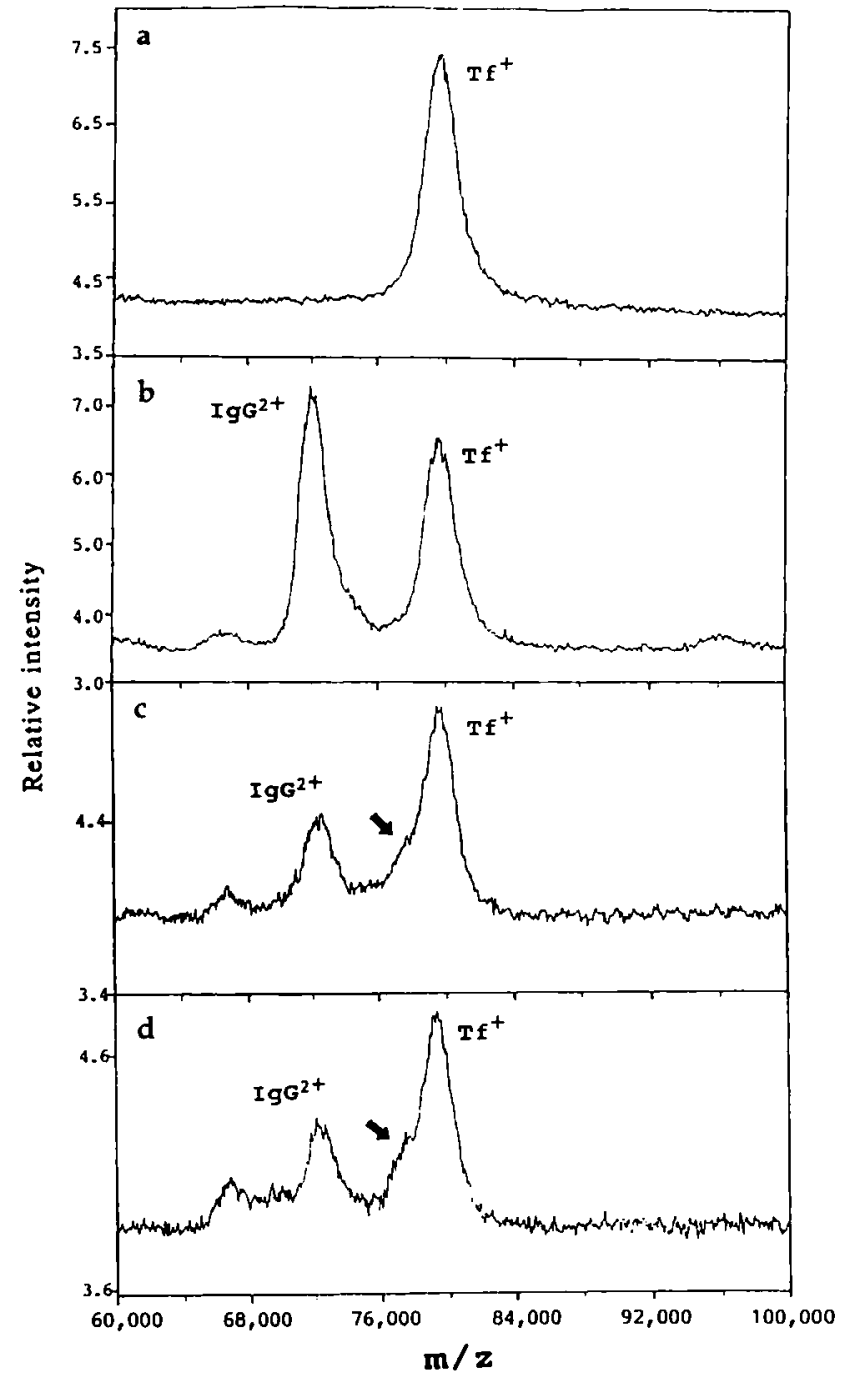

Figure 2. MALDI/TOF mass spectra of transferrin purified by affinity chromatography (a) and by immunoprecipitation (b-d). (a, b) normal control; (c) alcohol hard drinker; (d) CDG patient. Analyses used a JMS-LDI 1700. Arrows show additional ions on the low mass/charge shoulder of normal transferrin that are not seen in the control spectra.

be seen in the upper two control spectra. Similar spectra were observed in all analyzed samples of five heavy drinkers. In Figure 3, the MALDI/TOF mass spectra of the transferrin-antibody mixture from a CDG patient obtained with a linear type (LDI 1700) and a reflectron-type instrument (Vision 2000) are compared. Again, the region of the singly charged ion signal of transferrin is shown. In both cases, the shoulder on the low mass side of the normal transferrin signal that corresponds to the carbohydrate-deficient species is observed. However, a comparison between the obtained absolute molecular weights of the transferrin revealed reproducibly smaller values for both species in the reflectron than in the linear mode. Moreover, the molecular weights were much closer to the theoretical values in the linear mode.

In the next step, transferrin was treated with siali-

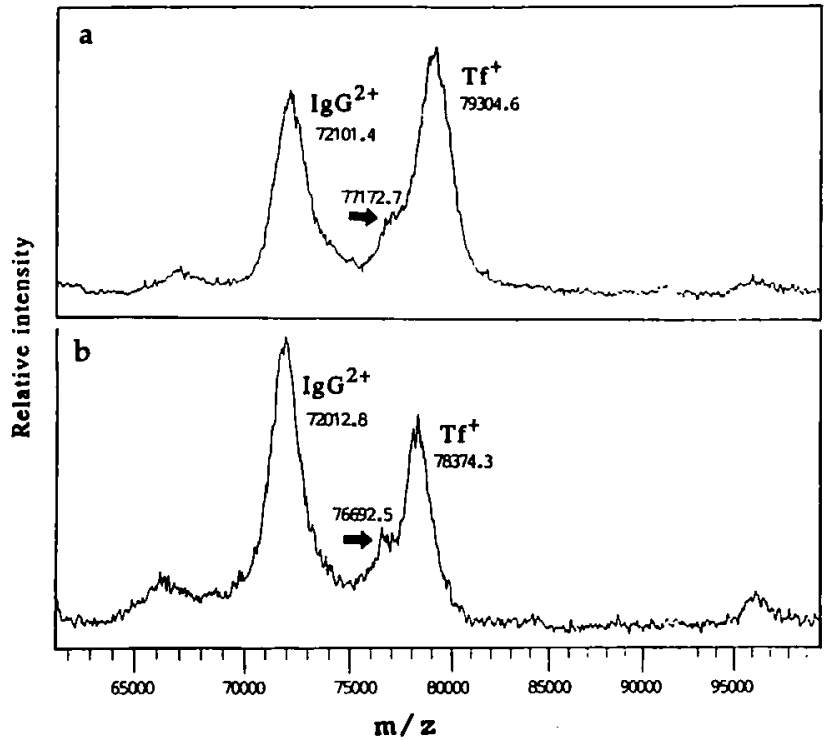

Figure 3. MALDI/TOF mass spectra of transferrin from a patient with CDG, analyzed by (a) linear type (LDI 1700) and (b) reflectron type MALDI/TOF mass spectrometry (Vision 2000). The region of the singly charged ion of transferrin is shown. The peak with the arrow is derived from carbohydrate-deficient transferrin.

dase to remove the sialic acids present on the oligosaccharide chain. Figure 4 shows the corresponding mass spectra of the intact and desialylated transferrin obtained by ESI and MALDI/TOF mass spectrometry. In ESI (Figure $4 a$ and $b$ ) as well in the linear mode MALDI (Figure $4 \mathrm{c}$ and d), the mass difference between the two samples was 1.1-1.2 ku, which corresponds to the mass of four sialic acids. In contrast, the reflectron mode MALDI spectra of both the native and the desialylated transferrin resulted in quite similar molecular weights (Figure $4 \mathrm{e}$ and $\mathrm{f}$ ). The obtained values correspond in both cases to the molecular weight of the desialylated transferrin. Table 1 gives an overview of all measured values. The values obtained by MALDI/ TOF mass spectrometry in Table 1 are the observed singly charged ion masses. The analyses were repeated several times and resulted in a reproducibility of about $0.35 \%$ (relative standard deviation) for the LDI 1700 and $0.1 \%$ for the Vision 2000 . By a comparison of the results in the linear and the reflectron modes, the content of the sialic acids can be estimated roughly, as discussed later. The average mass difference for the native normal transferrin obtained from the two modes is $1175 \mathrm{u}$. This fits quite well with the calculated mass of four sialic acids, which is $1165.2 \mathrm{u}$. Similarly, the loading values of sialic acids in the case of carbohydrate-deficient transferrin can be estimated roughly. Although the mass accuracy may be low because of the detection of the corresponding peak on the shoulder of the intact transferrin signal peak, the average mass difference between the carbohydrate-deficient transferrin signals in the linear and reflectron modes of about $790 \mathrm{u}$ is equivalent to 2.7 sialic acids. 

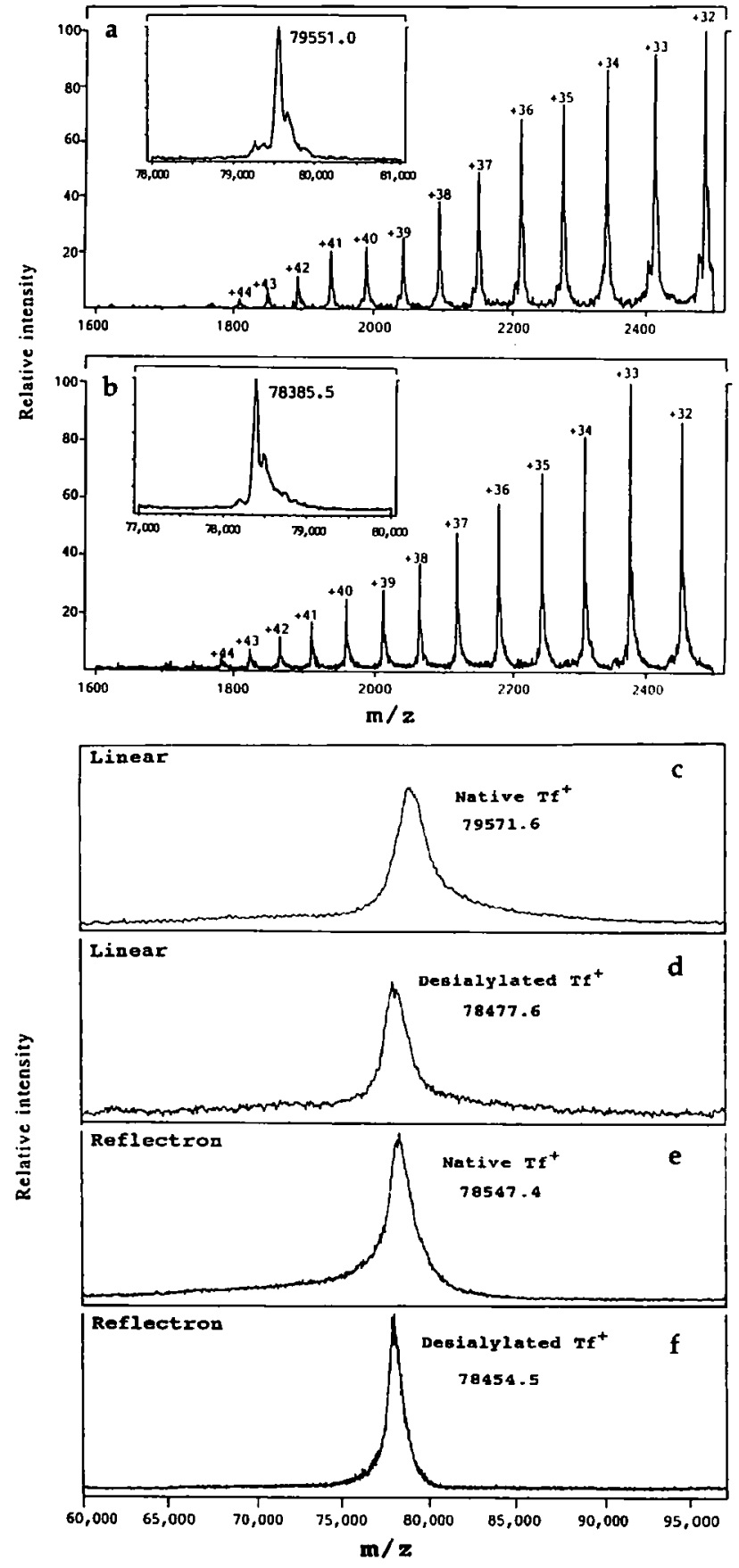

Figure 4. Comparison of mass spectra of intact and sialidasetreated transferrin obtained by MALDI/TOF mass spectrometry in the linear (LDI 1700) and the reflectron mode (Vision 2000) and by ESI-MS (TSQ-7000). Electrospray mass spectra and corresponding deconvoluted spectra are shown. The analysis was performed with microcapillary sample loading. Acetonitrile solution was used for elution. (a, b) ESI spectra; (c, d) linear mode MALDI/TOF mass spectra; $(e, f)$ reflectron mode MALDI/TOF mass spectrometry. (a, c, e) intact transferrin; (b, d, f) sialidasetreated transferrin.

\section{Discussion}

Serum transferrin precipitated by specific antibodies was successfully analyzed by MALDI/TOF and ESI mass spectrometry. Immunoprecipitates that contain transferrin and its antibody showed several ions by MALDI/TOF mass spectrometry, which correspond to transferrin, IgG, and other minor components. For ESIMS we first tried to load the immunoprecipitates through a conventional infusion pump, but no significant ion signals could be observed. In contrast, the spectra of immunoprecipitates analyzed by ESI-MS connected directly to a microcapillary reversed-phase column (on-line loading) showed clearly resolved ion signals. The better resolution by the on-line loading may be caused by the separation of transferrin and other proteins as well as the removal of the adducting species on the column. The adduct ions may be produced by contamination with substances in the glassware, water, reagents, fused silica, and dust from room air that bind to transferrin when loaded through the conventional infusion pump.

The purification procedure of affinity chromatography by using an immobilized specific antibody is rather complicated. It is time-consuming to isolate a specific antibody by using immobilized protein. For some substances it is difficult to obtain a sufficient amount of pure antigen to prepare the solid phase column. In contrast to affinity chromatography, the immunoprecipitation method does not require isolated specific antibody nor, therefore, pure antigen. The method requires only antiserum that contains a specific antibody against the protein to be tested, other antibodies, and all other serum components. However, when antiserum against transferrin was mixed with test serum, precipitates that were shown to contain only transferrin and IgG class antibody appeared. During mass spectrometry analysis, the signal due to $\mathrm{IgG}$ did not interfere with that of transferrin. Immunoprecipitation is a simple and rapid procedure and we believe it is suitable as a clinical laboratory test.

We analyzed transferrin prepared from patients with carbohydrate-deficient glycoprotein (CDG) syndrome. In ESI spectra of the main peak observed during microcapillary HPLC of immunoprecipitates from a normal control and a CDG patient, multiply charged ion peaks of normal transferrin were observed and the calculated molecular weight was consistent with the theoretical value. In the spectra from patient material the ion peaks of the subcomponent between the ion peaks of the main component were observed and the calculated molecular weight was $2.2 \mathrm{ku}$ less than the normal transferrin. This value corresponds to that of abnormal transferrin from CDG patient sera characterized by Yamashita et al. [7]. In the MALDI spectra of immunoprecipitate from CDG patient serum the ion peak on the shoulder peak of the normal component is also $2.2 \mathrm{ku}$ less than the normal molecule. The shoulder peaks also were reported by Wada et al. [10] in affinity purified transferrin from a CDG patient. When test serum was mixed with normal rabbit serum or purified normal immunoglobulin, no precipitate was obtained; accordingly no mass spectrometry signals were detected. The appearance of carbohydrate-defi- 
cient transferrin also has been reported in connection with regular high alcohol consumption [4]. That study revealed that the abnormal transferrin contains reduced proportions of the carbohydrates that constitute its terminal trisaccharide (sialic acid, galactose, and $\mathrm{N}$-acetylglucosamine). In our MALDI/TOF mass spectrometry analysis the transferrin spectra from heavy alcohol drinkers showed additional ions as a low mass/charge shoulder on the signal associated with normal transferrin. ESI-MS analyses of the same samples also revealed the variant molecule with almost the same molecular weight as the variant found in CDG syndrome patients. Inoue et al. [5] demonstrated by conventional chemical methods that transferrin from alcohol drinkers contains the molecule that lacks either or both of two $\mathrm{N}$-linked disialylated biantennary carbohydrate chains. Our findings are consistent with their evidence.

In MALDI/TOF mass spectrometry, the molecular weights obtained by the reflectron mode and the linear mode were different for the intact, sialic-acid containing transferrin. In the linear mode, it was in better agreement with the value achieved by ESI-MS and the theoretical value. The average mass difference in spectra of both modes corresponded to $4 \mathrm{~mol}$ of sialic acids, which is the actual number per molecule of intact transferrin. The observed molecular weight of the singly charged ion of intact transferrin measured by another reflectron MALDI instrument, the Kompact Maldi III, was $78.3-78.8 \mathrm{ku}$ [9], which is very close to that obtained by the Vision 2000 in the reflectron mode. However, the spectra of the desialylated transferrin showed no such mass difference. All these findings suggest that the bond of the sialic acids to the galactose is labile under MALDI conditions. The explanation of the difference in the molecular weight measured by linear and reflectron mode MALDI has been discussed [11, 12]. Most probably sufficient energy is stored in these bonds upon the desorption-ionization process to leave the glycoprotein ions in a metastable state. When the ions leave the source region, the sialic acids separate from the glycoprotein during flight through the field-free drift tube of the TOF mass spectrometer. After this process, termed "post source decay" $[16,17]$, the fragment ion will have less kinetic energy due to the loss in mass, but will retain its velocity (at least to first approximation). Therefore, in a linear mode TOF mass spectrometer where no additional accelerating fields influence the velocity of the ions, these fragments arrive at the detector at the same time as intact ions would, which results in a measurement of the mass of the original molecule even when it has decomposed. In contrast, a reflectron-type TOF mass spectrometer responds to the reduced kinetic energy of the ions. Here the fragments penetrate less deeply into the retarding reflectron field than intact ions with the correct kinetic energy; thus they return and arrive earlier at the detector. As long as the energy deficit is on the order of a few percent, the fragment ions can be focused quite correctly to their actual mass [18]. Therefore, a comparison between the results in both the linear and the reflectron mode can provide useful structural information due to an estimation of the number of sialic acids on the glycoprotein.

Immunoprecipitation is a suitable serum protein preparation method for mass spectrometry, especially for clinical diagnoses of protein abnormality. Polyclonal as well as monoclonal antibodies against many kinds of protein are commercially available. We believe that this procedure will provide a powerful strategy for the analysis of various kinds of protein in plasma, urine, and other body fluids.

\section{Acknowledgment}

The financial support of the Osaka Medical College Jinsenkai Foundation is gratefully acknowledged.

\section{References}

1. Meng, C. K.; Mann, M.; Fenn, J. B. Z. Phys. D 1988, 10, 361.

2. Hillenkamp, F.; Karas, M.; Beavis, R.; Chait, B. T. Anal. Chem. 1991, 63, 1193-1203A.

3. Jaeken, J.; van Eijck, H. G.; van der Heul, C.; Corbeal, L.; Eeckel, R.; Eggermont, E. Clin. Chim. Acto 1984, 144, 245-247.

4. Stibler, H.; Borg, S. In Biochemical and Social Aspects of Alcohol and Alcoholism; Kuriyama, K.; Takada, A.; Ishii, H., Eds.; Elsevier: Amsterdam, The Netherlands, 1988; pp 503-506.

5. Inoue, K.; Ookura, T.; Hukushima, K.; Maruyama, K.; Yuasa, I.; Yamashita, K. Seiknq 1993, 65, 1008(Abs).

6. Wada, Y.; Nishikawa, A.; Okamoto, N.; Inui, K.; Tsukamoto, H.; Okada, S.; Taniguchi, N. Biochem. Biophys. Res. Commun. 1992, 189, 832-836.

7. Yamashita, K.; Ideo, H.; Ohkura, T.; Fukushima, K.; Yuasa, I.; Ohno, K.; Takeshita, K. J. Biol. Chem. 1993, 268, 5783-5789.

8. Yamashita, K.; Ohkura, T.; Ideo, H.; Ohno, K.; Kanai, M. I. Biochem. 1993, 114, 766-769.

9. Nakanishi, T.; Okamoto, N.; Tanaka, K.; Shimizu, A. Biol. Mass Spectrom. 1994, 23, 230-233.

10. Wada, Y.; Gu, J.; Okamoto, N.; Inui, K. Biol. Mass Spectrom. 1994, 23, 108-109.

11. Huberty, M. C.; Vath, J. E.; Yu, W.; Martin, S. A. Anal. Chem. 1993, 65, 2791-2800.

12. Tsarbopoulos, A.; Karas, M.; Strupat, B. N.; Pramanik, T. L.; Nagabhushan, T. L.; Hillenkamp, F. Anal. Chem. 1994, 66, 2062-2070.

13. Okamoto, N.; Wada, Y.; Kobayashi, M.; Otani, K.; Tagawa, T.; Futagi, Y.; Imayoshi, Y.; Hayashi, A.; Shimizu, A.; Kato, Y. J. Inher. Metab. Dis. 1993, 16, 435-440.

14. Boemsen, K. O.; Schaer, M.; Widmer, H. M. Chimica 1990, 44 , 412-416.

15. Ingendoh, A.; Karas, M.; Hillenkamp, F.; Giessmann, U. Int. I. Mass Spectrom. Ion Processes 1994, 131, 345-354.

16. Spengler, B.; Kirsch, D.; Kaufmann, R.; Jaeger, E. Rapid Commun. Mass Spectrom., 1992, 6, 105-108.

17. Kaufmann, R.; Kirsch, D.; Spengler, B. Int. J. Mass Spectrom. Ion Processes 1994, 131, 355-386.

18. Boesl, U.; Winkauf, R.; Schiag, E. W. Int. J. Mass Spectrom. Ion Processes 1992, 112, 121-166. 